\title{
Cardinal roles of miRNA in cardiac development and disease
}

\author{
FENG YuLiang $^{1,2} \&$ YU XiYong $^{1 *}$ \\ ${ }^{1}$ Medical Research Center of Guangdong General Hospital, Guangdong Academy of Medical Sciences, Guangzhou 510080, China: \\ ${ }^{2}$ Southern Medical University Pharmaceutical School, Guangzhou 510515, China
}

Received October 11, 2011; accepted October 31, 2011

\begin{abstract}
MicroRNAs (miRNAs) are small noncoding RNAs that are emerging as pivotal modulators in virtually all aspects of cardiac biology, from cardiac development to cardiomyocyte survival and hypertrophy. The miRNA profiling, following gain- and loss-of-function studies using in vitro and in vivo models, has identified wide-ranging functions for miRNAs in the heart, providing new perspectives on their contributions to cardiac pathogenesis, and revealing potential therapeutic targets and diagnostic biomarkers. This review summarizes current progress in regulation of miRNAs in heart development and disease.
\end{abstract}

miRNA, cardiac, development, disease

Citation: Feng Y L, Yu X Y. Cardinal roles of miRNA in cardiac development and disease. Sci China Life Sci, 2011, 54: 1113-1120, doi: $10.1007 / \mathrm{s} 11427-011-4257-8$

MicroRNAs (miRNAs) are endogenous small non-coding RNAs of approximately 22 nucleotides, which are processed from one arm of stem-loop hairpin RNA precursors by the action of Dicer, a ribonuclease III-like enzyme [1]. miRNAs play an important regulatory role in animals and plants, resulting in gene silencing via inhibition of translation [2] and mRNA degradation [3]. It has been estimated that the human genome can encode up to 1000 miRNAs, and to date, hundreds have been experimentally identified [1]. Each miRNA can target multiple mRNA sites, with several hundred targets per miRNA possible, thereby enabling modulation of gene expression patterns rather than a single gene. miRNAs have been shown to play a role in the regulation of development, tissue homeostasis and disease pathogenesis [4].

\section{1 miRNA in cardiac development}

A large set of miRNAs expressed in the heart have been identified by direct cloning [5] and microarray-based pro-

*Corresponding author (email: yuxycn@ hotmail.com) filing [6-9], with miR-1 and miR-133 found to be highly enriched in cardiac and skeletal muscle. miR-1 is most abundant in heart, representing $45 \%$ of total miRNAs in the adult mouse heart and $24 \%$ in the adult human heart. Its expression coincides with the onset of cardiomyocyte differentiation in the developing heart tube, and somitic myotome development in chick embryos [10] and the looping mouse heart at embryonic day (E) 8.5, where it is regulated by SRF (serum response factor) [11]. Two members of the miR-1 class of RNAs, miR-1-1 and miR-1-2, are specifically expressed in cardiac tissue and skeletal muscle. miR-1-1 and miR-1-2 are encoded separately by different chromosomes but appear to target the same mRNAs. To determine the particular role of miR-1-2, Zhao et al. [11] used a targeted deletion strategy to eliminate the miR-1-2 locus without affecting adjacent genes. Approximately 50\% of mice lacking miR-1-2 died during embryonic development, showing abnormal morphogenesis, including ventricular septal defects [12].

In Drosophila, dmiR-1 is expressed in the mesoderm during embryonic development (stage 5) and subsequently in all its muscle cell derivates including the dorsal vessel 
[13]. Using a conditional knockout strategy, dmiR-1 was shown to be critical for muscle development [13]. Conversely, in rodent heart, miR-1 is essential for cardiogenesis, with overexpression of miR-1 in a transgenic model driven by the beta myosin heavy chain ( $\beta$-MHC) promoter, leading to developmental arrest at E13.5.

miR-1 targets Hand2, a transcription factor responsible for the regulation of embryonic cardiac ventricle expansion; therefore it is feasible that the phenotype observed in embryonic rodent heart is due to the reduction of endogenous Hand 2 by excess miR-1 [11]. Furthermore, miR-1 functions have been addressed using singular genomic ablation strategies. The resulting homozygous knockout mice were lethal in development (E15.5) and early neonatal life, with no observed effect on the survival of heterozygous animals [12], likely because of compensation for miR-1 reduction by the second gene. Ventricular septal defect was the leading cause of lethality in half of homozygous embryos. Of the remaining mice, $15 \%$ survived early lethality; however, wall thickening due to hyperplasia, accompanied by prolongation of the action potential and occasional sudden death, was observed in these animals. This may be explained by the reduction in Irx5, a negative transcriptional regulator of the potassium channel Kcnd2/Kv4.2, by excess miR-1 [12], leading to repolarization defects and subsequent electrical conduction abnormality [14].

miR-133 is another muscle-enriched miRNA, although its abundance in heart is less than miR-1. In contrast to miR-1, with deletion of one or two miR-1 genes resulting in lethality in knockout mice, deletion of one heart expressed gene of miR-133 had no effect on heart development. Following targeted deletion of miR-133a-1 or miR-133a-2, no obvious cardiac morphological and functional abnormalities were observed, likely because of the redundant action of the genes, with each gene accounting for approximately $50 \%$ of function [15]. However, combined targeted deletion of miR-133a-1 and miR-133a-2 resulted in embryonic and postnatal lethality. This early mortality correlated with ventricular septal defects and enlargement of the right ventricle and atria due to development of dilated cardiomyopathy and increased fibrosis [15].

The phenotypic abnormalities of the miR-133a-1/miR133a-2 knockout mice result from the upregulation of SRF and cyclin D2, two of the downstream targets of miR-133, leading to ectopic expression of smooth muscle genes in heart tissue, aberrant cardiomyocyte proliferation and apoptosis. Moreover, transgenic miR-133a overexpression in heart resulted in thinning of ventricular walls, an insufficient pumping action of the heart and embryonic lethality (E15.5) [15] (Figure 1).

\section{2 miRNA in heart disease}

\subsection{Cardiac hypertrophy}

There are two forms of cardiac hypertrophy: (i) physiologi- cal, the normal response to healthy exercise or pregnancy which results in an increase in myocardial mass and pumping ability; (ii) pathological, the size of the heart initially increases to compensate for damage to the heart due to metabolic abnormality, stress or disease, but subsequently leads to a decline in left ventricular function [16]. Pathological hypertrophy is associated with all forms of heart failure observed in the clinical setting. Hence, to understand its underlying mechanism is of great importance for drug development to cure cardiac disease. Pathological hypertrophy is mainly caused by hypertension, loss of myocytes subsequent to myocardial infarction (MI) or ischemic/reperfusion ( $\mathrm{I} / \mathrm{R})$ injury, and genetic alterations that lead to enlargement of the left ventricles, such as dilated cardiomyopathy. Moreover, metabolic abnormality or stress can also lead to hypertrophy [17]. Currently, there is great interest in determining the molecular mechanisms, including the roles of miRNAs, in pathological hypertrophy (Figure 1). Using animal models, genome wide miRNA microarrays have been used to isolate miRNAs which are upregulated, downregulated or remain unchanged, in cardiac hypertrophy compared with a normal heart [18-24]. From these studies, certain miRNAs are more frequently reported by different research groups, indicating the possibility that these miRNAs might have common roles in hypertrophy pathogenesis. Among the frequently reported miRNAs, miR-21, miR-23a, miR-24, miR-125, miR-129, miR-195, miR-199, miR-208 and miR-212 are often upregulated with hypertrophy, whereas miR-1, miR-133, miR-29, miR-30 and miR-150 are often downregulated. Interestingly, transgenic overexpression of individual miRNAs, including miR-23a, miR-23b, miR-24, miR-195, miR-199a and miR-214, all upregulated with cardiac hypertrophy, was sufficient to induce cardiac hypertrophy. Furthermore, in a knock-in mouse model, overexpression of miR-195 was sufficient to drive pathological cardiac growth [18]. By contrast, in vitro overexpression of miR-150 and miR-181b, both downregulated in cardiac hypertrophy, resulted in reversal of cardiomyocyte size [18]. The ability of individual miRNAs to mimic cardiac phenotypes implies that the different miRNA is a cause rather than simply a consequence of pathologic cardiac remodeling.

Although high-throughput miRNA microarray or RNA sequencing have identified a set of miRNAs involved in cardiac hypertrophy, the transcriptional mechanism that regulates the expression of miRNAs responsible for cardiac hypertrophy needs further investigation. Transcriptional regulation of miRNAs is well studied for miR-1/miR-133. $\mathrm{SRF}$ is a cardiac-enriched transcription factor responsible for the regulation of organized sarcomeres in the heart [25]. SRF interacts synergistically with myocardin to activate miR-1-1 and miR-1-2 by binding to the upstream SRFbinding consensus element CArG box [11]. Myocyte enhancer factor-2 (MEF2) also activates transcription of the 


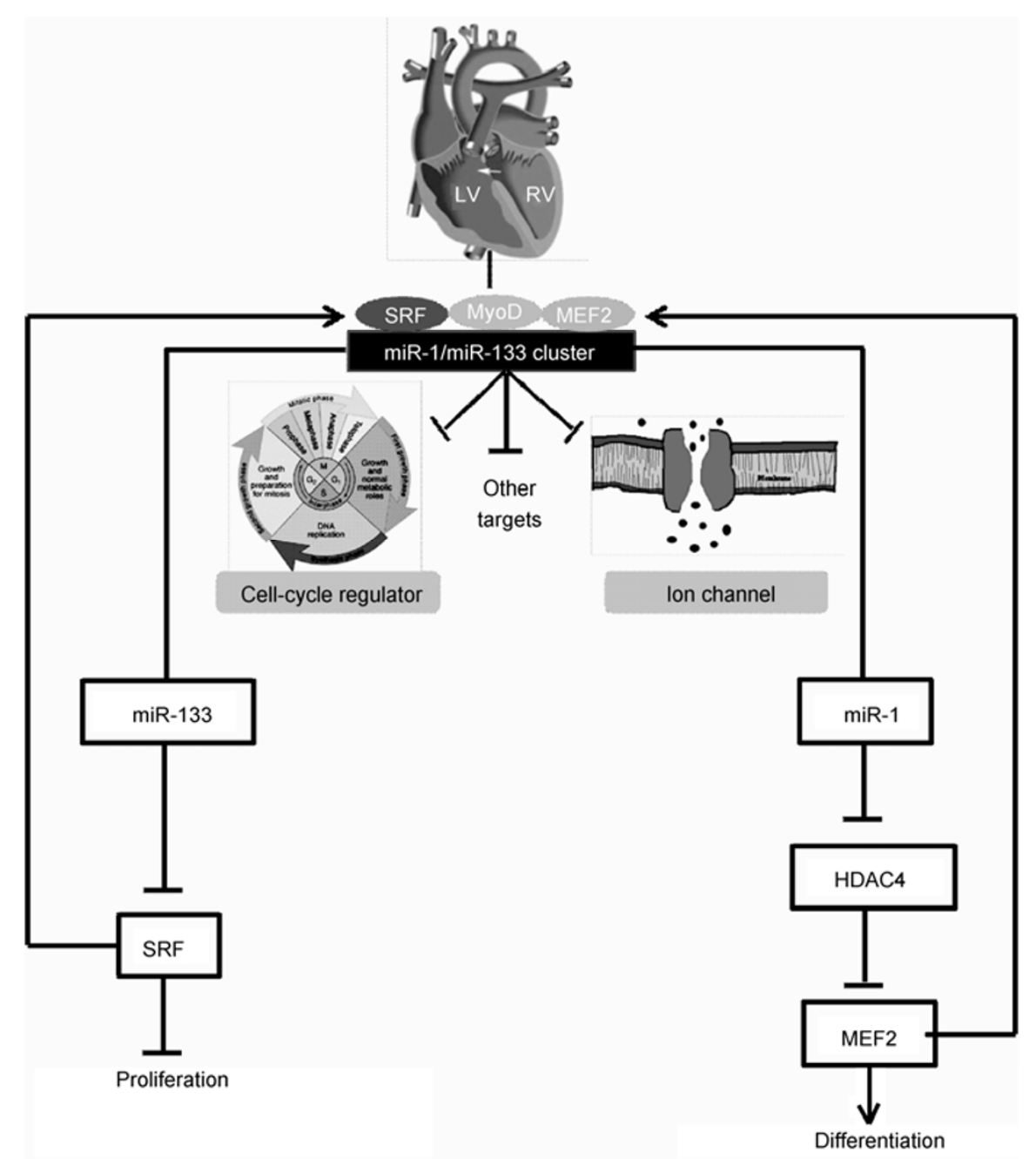

Figure 1 Critical roles of the miR-1/miR-133 cluster in muscle development. The tissue-specific expression of miR-1 and miR-133 clusters is driven by the transcription factors SRF, MEF2 and MyoD. miR-1 promotes muscle differentiation by repressing the expression of HDAC4 (histone deacetylase 4). HDAC4, in turn, binds and inhibits activation of the critical muscle transcription factor, MEF2 (myocyte enhancer factor-2), which potently activates the expression of myoblast differentiation genes and miR-1. miR-133 causes downregulation of SRF, a crucial regulator of muscle differentiation, and enhances the proliferation of myoblasts, inhibiting their differentiation.

bicistronic precursor RNA encoding miR-1-2 and miR133a-1, via an intragenic muscle-specific enhancer [26]. It was reported that nuclear factor of activated $\mathrm{T}$ cells isoform 3 (NFATc3), well known as a key factor in mediating the hypertrophic signal of calcineurin [27], transactivates the expression of miR-23a by binding directly to the promoter region of miR-23a, which subsequently suppresses translation of muscle specific zinc finger protein 1 [28]. miR-1 negatively regulates the expression of hypertrophy-associated calmodulin, MEF2a and GATA4, attenuating calcium-dependent signaling through the calcineurin-NFAT pathway [29]. Recently, da Costa Martins et al. demonstrated that miR-199b is a direct calcineurin/NFAT target gene, which increases in expression in mouse and human heart failure. Additionally, mutant mice overexpressing miR-199b, or haploinsufficient for Dyrk1a, are sensitized to calcineurin/NFAT signaling or pressure over- load, and show stress-induced cardiomegaly through reduced Dyrk1a expression, implicating miR-199b as a therapeutic target in cardiac hypertrophy [30].

Moreover, it appears that different miRNAs have distinct mechanisms in regulating hypertrophy by the targeting of different targets. miR-133 inhibits hypertrophy through targeting RhoA and Cdc42 [31]. It was reported that targets of miR-208 include thyroid hormone receptor-associated protein 1 , suggesting that miR-208 initiates cardiomyocyte hypertrophy by regulating triiodothyronine-dependent repression of the $\beta$-myosin heavy chain $[32,33]$. miR-27a also regulates $\beta$-myosin heavy chain gene expression by targeting TRb1 in cardiomyocytes [34]. It is clear that excessive reactive oxygen species (ROS) play a critical role in the development of cardiac hypertrophy. In contrast, antioxidants scavenge ROS, thereby maintaining the reduced environment of cells and inhibiting hypertrophy in the heart. 
Thioredoxin1 (Trx1) has been shown to function as a major antioxidant in the heart and to interact with important signaling molecules and transcription factors, thereby attenuating cardiac hypertrophy [35]. Nevertheless, the signaling pathway of Trx1 mediated anti-hypertrophy remains to be fully elucidated. Recently, the Sadoshima lab demonstrated the involvement of the let-7 family, including miR-98, in Trx1 inhibition of cardiac hypertrophy, induced by angiotensin II via downregulation of cyclin D2 [36].

\subsection{Ischemic cardiomyopathy}

Cardiomyocyte death, or apoptosis, is a key cellular event in ischemic cardiomyopathy [37]. It is well established that multiple genes are aberrantly expressed in infarcted hearts, and are responsible for cardiac remodeling following MI or $\mathrm{I} / \mathrm{R}$ injury [38]. Therefore, it is reasonable to hypothesize that miRNAs could be involved in ischemic cardiomyopathy (Figure 2). Recently, Ren et al. conducted a novel study using a mouse model of cardiac I/R in vivo and ex vivo, to determine the miRNA signature in ischemic hearts, and found that miR-320 expression was consistently dysregulated following ischemia [39]. They also identified heat-shock protein 20 (Hsp20), a known cardioprotective protein, as a target of miR-320. Knockdown of endogenous miR-320 produced a protective effect against I/R induced cell death, by targeting of Hsp20. Further studies from the same group showed that the miR-144/451 cluster, regulated by GATA4, also provides protection against simulated I/R-induced cardiomyocyte death via targeting of the CUGBP2-COX-2 pathway. This suggests that GATA4 is not only involved in cardiogenesis but myocardial survival [40] (Figure 3).

Investigation of the miRNA expression pattern rat hearts at $6 \mathrm{~h}$ following MI revealed that miR-21 expression was significantly downregulated in infarcted areas but upregulated in border areas [41]. Adenoviral transfer of miR-21 in vivo decreased cell apoptosis in the border and infarcted areas through its target gene, programmed cell death 4 , and the activator protein 1 pathway. In vitro experiments showed that miR-1 and miR-133 produced opposing effects on apoptosis induced by oxidative stress in H9c2 rat ventricular cells, with miR-1 being pro-apoptotic and miR-133 being anti-apoptotic. Post-transcriptional repression of HSP60 and HSP70 by miR-1 and of caspase- 9 by miR-13, contributes significantly to their opposing actions. miR- 1 is also associated with the cell death pathway by inhibiting translation of insulin-like growth factor-1 $[42,43]$.

Early ischemia or hypoxia preconditioning is an immediate cellular reaction to brief hypoxia/reoxygenation cycles that involves de novo protein synthesis, but not mRNA synthesis [44]. It is a mechanism required to protect the heart against subsequent prolonged ischemia or I/R induced damage [45]. The miR-29 family, which is downregulated after myocardial infarction, inhibits the expression of several collagens and extracellular matrix proteins, thereby contributing to scar formation and fibrosis. Similarly, the miR-199 family is rapidly downregulated in cardiac myocytes under hypoxic conditions, relieving the repression of sirtuin 1 (SIRT1) and hypoxia-inducible factor $1 \alpha(\mathrm{HIF}-1 \alpha)$ in a model of hypoxia preconditioning [46], suggesting that miR-199a directly targets and inhibits translation of

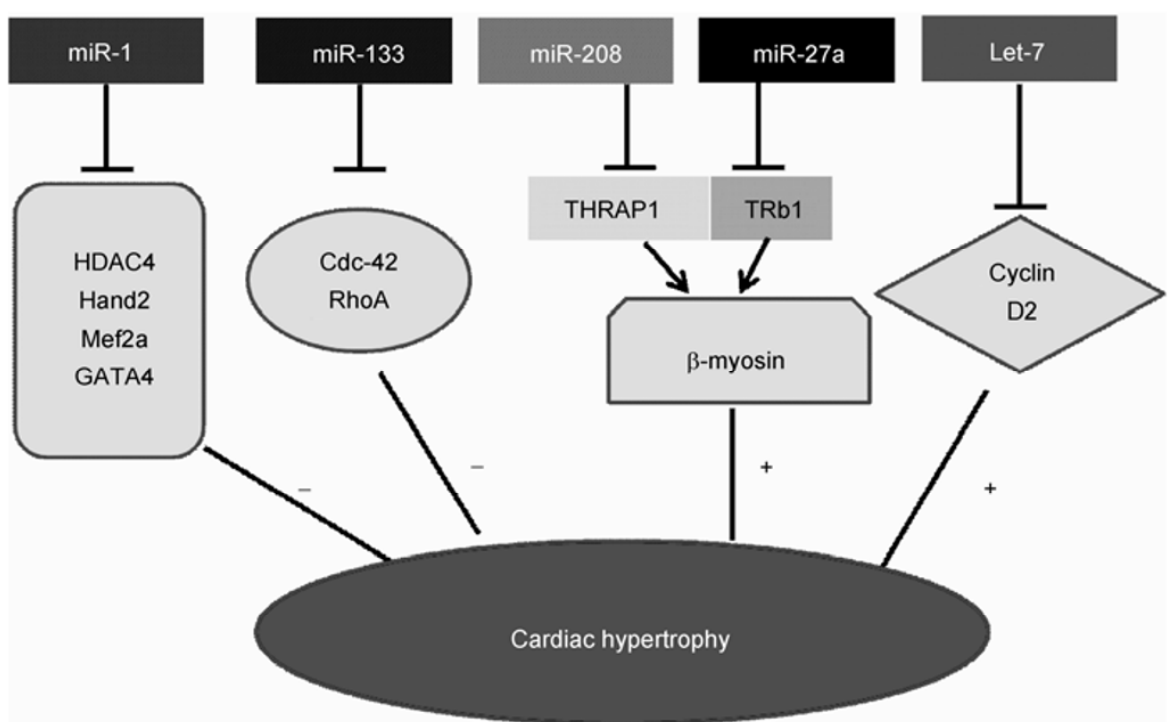

Figure 2 miRNAs and target genes involved in cardiac hypertrophy. Upregulation or downregulation of a specific miRNA is represented by an upward or a downward arrow, respectively. Expression level changes in target genes inversely correlate with that of the targeting miRNA. The targets shown are histone deacetylase 4 (HDAC4), heart- and neural crest derivatives-expressed protein 2 (HAND2), myocyte-specific enhancer factor 2C (mef2c), GATA binding protein 4 (GATA4), cell division control protein 42 homolog (cdc-42), ras homolog gene family member A (RhoA), thyroid hormone receptor associated protein 1 (THRAP1), telomere repeat binding factor 1 (TRB1) and cyclin D2. 


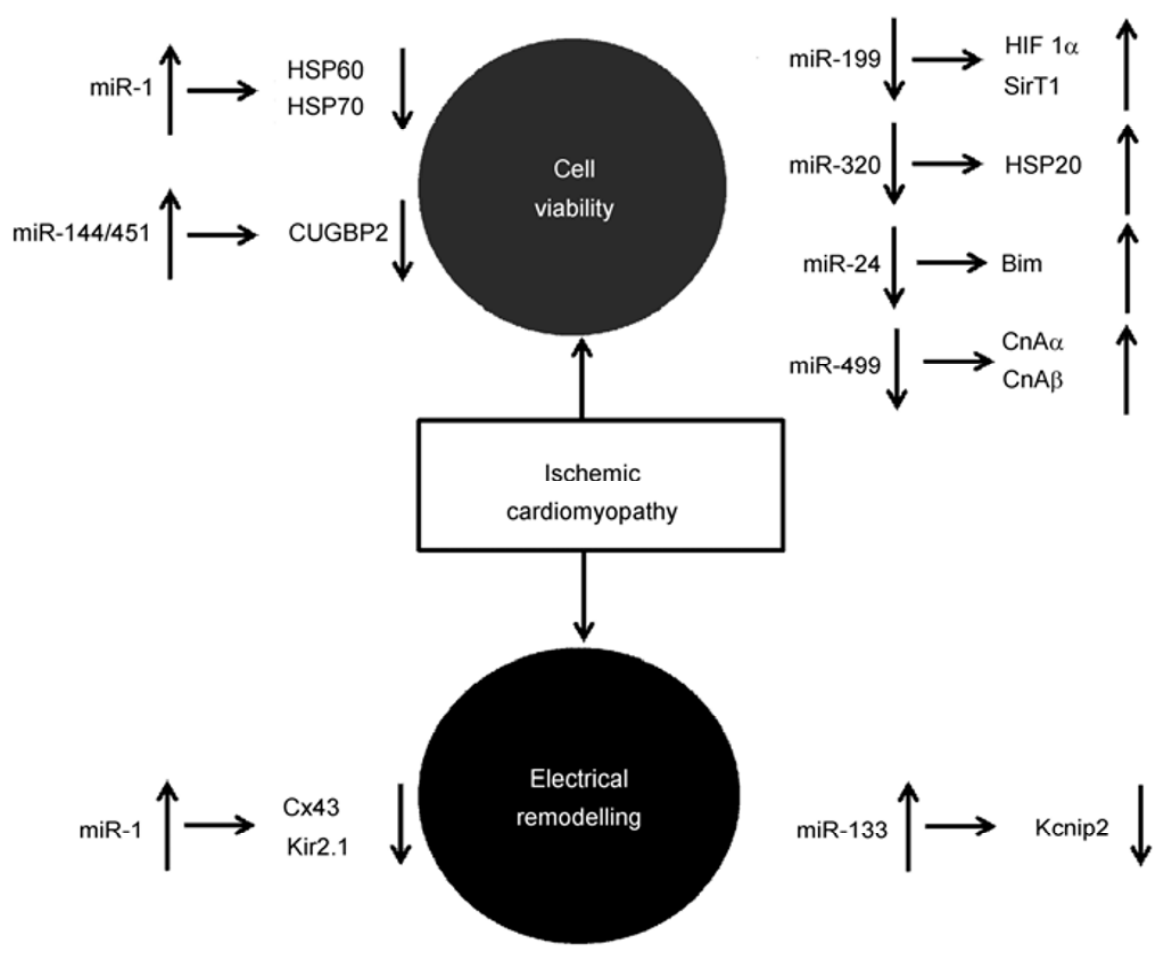

Figure 3 miRNAs and target genes involved in ischemic cardiomyopathy. The different miRNAs and their targets, involved in cell viability and electrical remodeling during ischemic cardiomyopathy. The targets shown are hypoxia-inducible factor 1 (Hif-1), bcl-2 interacting protein (Bim), calcineurin A-alpha (CnA-alpha), calcineurin A-beta (CnA-beta), CUG-BP- and ETR-3-like factor 2 (CUGBP2), sirtuin 1 (SirT1), heat shock proteins 20, 60, and 70 (HSP20, HSP60, HSP70), caspase 9, connexin 43 (Cx43), inward rectifier potassium channel 2 (Kir2.1) and cardiac voltage-gated potassium channel modulatory subunit (Kcnip2).

HIF-1 $\alpha$ and SIRT1. HIF-1 $\alpha$ is a master regulator of hypoxia-induced gene transcription, and is regulated by a post-transcriptional oxygen-sensitive mechanism that triggers its immediate expression subsequent to a drop in oxygen levels. Therefore, miR-199a is likely a key modulator of a hypoxia-triggered pathway and an ideal molecule for preconditioning the cells against hypoxic insult. Since it has been reported that SIRT1 was upregulated, leading to deacetylation and inactivation of HIF-1 $\alpha$ in hypoxia [47], it would be interesting to examine the crosstalk between miR-199/SIRT1/HIF1a.

Recently, the Srivastava Deepark lab reported that miRNA-24 expression is downregulated in the ischemic border zone of the murine left ventricle following MI [48]. miR-24 suppresses cardiomyocyte apoptosis, in part by direct repression of Bim, the BH3-only domain-containing protein, which positively regulates apoptosis. In vivo expression of miR-24 in a mouse MI model inhibited cardiomyocyte apoptosis, attenuated infarct size and reduced cardiac dysfunction. This antiapoptotic effect on cardiomyocytes in vivo was partially mediated by Bim. Thus manipulating miRNA levels during stress-induced apoptosis may be a novel therapeutic strategy for ischemic cardiomyopathy.

Other groups have reported that modulation of miR-499 levels affects apoptosis as well as the severity of myocardial infarction and cardiac dysfunction induced by I/R [49]. These studies show that the $\alpha$ - and $\beta$-isoforms of the calcineurin catalytic subunit are direct targets of miR-499, and that miR-499 inhibits cardiomyocyte apoptosis through its suppression of calcineurin-mediated dephosphorylation of dynamin-related protein-1 (Drp1), thereby decreasing Drp1 accumulation in mitochondria and Drp1-mediated activation of the mitochondrial fission program. Furthermore, they found that p53 negatively regulated miR-499 transcription, suggesting an interaction between p53, miR-499, calcineurin and Drp1 in the apoptotic program of the heart. Modulation of miR-499 may represent a therapeutic approach to treat apoptosis-related cardiac disease, including myocardial infarction [49].

\subsection{Arrhythmia}

A major goal for cardiovascular medicine is to develop improved approaches to prevent electrical remodeling and arrhythmias, which are characteristic of ischemic heart disease [50,51].

As miRNAs regulate the cells response to ischemia, they also control the expression of molecules involved in regulating action potentials and cardiac conduction. miR-1 is upregulated in the heart from patients with coronary artery disease (CAD) and in rat ischemic hearts, which correlates 
to an increase in arrhythmogenesis [52]. Gain-of-function or loss-of-function of miR-1 in ischemic rat heart leads to an equivalent effect on the frequency of arrhythmias. miR-1 targets the gap junction proteins $\mathrm{Cx} 43$ and Kir2.1, a subunit of the $\mathrm{K}^{+}$channel that mediates $I_{\mathrm{K} 1}$. Knockdown of endogenous miR-1 could therefore inhibit arrhythmias, whereas concomitant knockdown of both $\mathrm{Cx} 43$ and Kir2.1 may reverse this effect. In support of this, genomic ablation of Cx43 [53], or missense mutation or $I_{\mathrm{K} 1}$ [54], caused increased episodes of arrhythmias. Interestingly, in contrast to their regulation in ischemic conditions, miR-1 and miR-133 are decreased during cardiac hypertrophy, with miR-1 targeting $\mathrm{HCN} 2$ and $\mathrm{HCN} 4$, and miR-133 targeting $\mathrm{HCN} 2$ [55]. These proteins are key components of the pacemaker channel involved in the hyperpolarization-activated current $\left(I_{\mathrm{f}}\right)$. They are also upregulated during cardiac hypertrophy and failure, and have been implicated in arrhythmogenesis [56]. The downregulation of miR-133 during cardiac hyper-

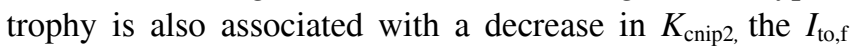
accessory subunit, albeit via an indirect mechanism, and is accordingly, responsible for prolongation of the QT interval [57]. Taken together, miR-1 and miR-133 are multifaceted molecules involved in hypertrophic and ischemic heart by targeting of a spectrum of mRNAs.

Recently, it was reported that miR-328 contributes to adverse electrical remodeling in atrial fibrillation (AF), partially through targeting L-type $\mathrm{Ca}^{2+}$ channel genes. This provides a novel molecular mechanism for AF and implicates miR-328 as a potential therapeutic target for AF [58].

\section{3 miRNAs as diagnostic tools for cardiac di- sease}

Recent studies have shown that some miRNAs are present in circulating blood delivered by exosomes and microparticles $[59,60]$. Circulating miRNAs have emerged as useful diagnostic tools in several diseases [61-64]. In cardiovascular disease, circulating miR-208 has been shown in a rodent model of myocardial injury [65]. Additionally, circulating miRNAs have been reported in patients with myocardial infarction [66]. Accordingly, it has been hypothesized that miRNAs in the systemic circulation reflect tissue damage, and can be used as biomarkers of myocardial infarction [67-69].

Li et al. demonstrated for the first time, a circulating miRNA profile for hypertensive patients, and revealed a novel link between HCMV infection and essential hypertension [70]. These findings provide important insights into the pathogenesis of essential hypertension.

Furthermore, a recent report suggests serum levels of cardiac-expressed miRNAs react to cardiac injury in a manner similar to that of cardiac enzymes. The plasma levels of miRNAs-208b and -499 have been shown to increase by more than 1000 -fold after myocardial infarction, mirroring the troponin T levels [71]. Viral myocarditis was associated with 6- to 30-fold increases in miRNA-499, whereas acute heart failure showed only a 2-fold increase. Likewise, circulating levels of cell-free miRNA-1 were significantly increased in patients with acute myocardial infarction (AMI), which positively correlated with serum CK-MB levels [72]. Another clinical study showed that miRNA-1, miRNA-133a and miRNA-208a in blood from patients with AMI were elevated compared with those from patients without AMI. Despite these encouraging results, the number of samples in the studies is too small to provide definite proof of the diagnostic power of miRNA signatures and their value for clinical testing of AMI patients. Therefore, future prospective trials on large patient cohorts are needed to establish miRNAs as a novel biomarker class for AMI. Recently, it was reported that elevated levels of circulating miR-1 and miR-133a in patients with cardiovascular disease originate mainly from the injured myocardium, indicating miR-1 and miR-133 in serum may serve as biomarkers for myocardial damage [73].

\section{Future perspectives}

Recent studies provide clear evidence that miRNAs modulate a diverse spectrum of cardiac functions with developmental, pathophysiological and clinical implications; however, many gaps remain in our understanding of miRNA-based regulation of gene expression in the normal and diseased heart. For example, it will be a great challenge to differentiate the mRNAs that are truly relevant to a particular miRNA-regulated process, since one miRNA may target many mRNAs. To aid elucidation of this problem is the apparent variability in miRNA function based on physiological context or cell type, making it necessary to define the potentially different functions of individual miRNAs in different settings. Another important consideration is that interactions between multiple miRNAs with common target mRNAs are likely to play a major role in gene regulation. Thus, it will be of importance to identify sets of miRNAs acting cooperatively within the heart. This has significant implications in development of miRNA-based therapeutics, as administration of miRNA inhibitor cocktails may prove more efficacious than targeting a single miRNA. Notwithstanding these and other hurdles, the rapid technical advances we are currently witnessing, and the insight that is being derived from application of these methods, engenders enthusiasm that miRs will lead to "miRacles" rather than a "miRage".

This work was supported by the National Natural Science Foundation of China (Grant No. 81070103) and National Natural Science Foundation of Major International Cooperation Projects in China (Grant No. 81120108003) to Yu XiYong. 
1 De Rosa S, Fichtlscherer S, Lehmann R, et al. Transcoronary concentration gradients of circulating microRNAs. Circulation, 2011, 124: 1936-1944

2 Kiriakidou M, Tan G S, Lamprinaki S, et al. An mRNA m7G cap binding-like motif within human Ago2 represses translation. Cell, 2007, 129: 1141-1151

3 Bagga S, Bracht J, Hunter S, et al. Regulation by let-7 and lin-4 miRNAs results in target mRNA degradation. Cell, 2005, 122: 553-563

4 Bauersachs J, Thum T. Biogenesis and regulation of cardiovascular microRNAs. Circ Res, 2011, 109: 334-347

5 Lagos-Quintana M, Rauhut R, Yalcin A, et al. Identification of tissue-specific microRNAs from mouse. Curr Biol, 2002, 12: 735-739

6 Chendrimada T P, Gregory R I, Kumaraswamy E, et al. TRBP recruits the Dicer complex to Ago2 for microRNA processing and gene silencing. Nature, 2005, 436: 740-744

7 Sayed D, Hong C, Chen I Y, et al. MicroRNAs play an essential role in the development of cardiac hypertrophy. Circ Res, 2007, 100: 416-424

8 Tatsuguchi M, Seok H Y, Callis T E, et al. Expression of microRNAs is dynamically regulated during cardiomyocyte hypertrophy. J Mol Cell Cardiol, 2007, 42: 1137-1141

9 van Rooij E, Sutherland L B, Liu N, et al. A signature pattern of stress-responsive microRNAs that can evoke cardiac hypertrophy and heart failure. Proc Natl Acad Sci USA, 2006, 103: 18255-18260

10 Darnell D K, Kaur S, Stanislaw S, et al. MicroRNA expression during chick embryo development. Dev Dyn, 2006, 235: 3156-3165

11 Zhao Y, Samal E, Srivastava D. Serum response factor regulates a muscle-specific microRNA that targets Hand2 during cardiogenesis. Nature, 2005, 436: 214-220

12 Zhao Y, Ransom J F, Li A, et al. Dysregulation of cardiogenesis, cardiac conduction, and cell cycle in mice lacking miRNA-1-2. Cell, 2007, 129: 303-317

13 Sokol N S, Ambros V. Mesodermally expressed Drosophila microRNA-1 is regulated by Twist and is required in muscles during larval growth. Genes Dev, 2005, 19: 2343-2354

14 Costantini D L, Arruda E P, Agarwal P, et al. The homeodomain transcription factor Irx 5 establishes the mouse cardiac ventricular repolarization gradient. Cell, 2005, 123: 347-358

15 Liu N, Bezprozvannaya S, Williams A H, et al. microRNA-133a regulates cardiomyocyte proliferation and suppresses smooth muscle gene expression in the heart. Genes Dev, 2008, 22: 3242-3254

16 Levy D, Garrison R J, Savage D D, et al. Prognostic implications of echocardiographically determined left ventricular mass in the Framingham Heart Study. N Engl J Med, 1990, 322: 1561-1566

17 Rajabi M, Kassiotis C, Razeghi P, et al. Return to the fetal gene program protects the stressed heart: a strong hypothesis. Heart Fail Rev, 2007, 12: 331-343

18 van Rooij E, Sutherland L B, Liu N, et al. A signature pattern of stress-responsive microRNAs that can evoke cardiac hypertrophy and heart failure. Proc Natl Acad Sci USA, 2006, 103: 18255-18260

19 Chen J F, Murchison E P, Tang R, et al. Targeted deletion of Dicer in the heart leads to dilated cardiomyopathy and heart failure. Proc Natl Acad Sci USA, 2008, 105: 2111-2116

20 Cheng Y, Ji R, Yue J, et al. MicroRNAs are aberrantly expressed in hypertrophic heart: do they play a role in cardiac hypertrophy? Am J Pathol, 2007, 170: 1831-1840

21 Sayed D, Hong C, Chen I Y, et al. MicroRNAs play an essential role in the development of cardiac hypertrophy. Circ Res, 2007, 100: $416-424$

22 Ikeda S, Kong S W, Lu J, et al. Altered microRNA expression in human heart disease. Physiol Genomics, 2007, 31: 367-373

23 Tatsuguchi M, Seok H Y, Callis T E, et al. Expression of microRNAs is dynamically regulated during cardiomyocyte hypertrophy. J Mol Cell Cardiol, 2007, 42: 1137-1141

24 Thum T, Galuppo P, Wolf C, et al. MicroRNAs in the human heart: a clue to fetal gene reprogramming in heart failure. Circulation, 2007, 116: $258-267$

25 Olson E N, Schneider M D. Sizing up the heart: development redux in disease. Genes Dev, 2003, 17: 1937-1956

26 Tay Y, Zhang J, Thomson A M, et al. MicroRNAs to Nanog, Oct4 and Sox 2 coding regions modulate embryonic stem cell differentiation. Nature, 2008, 455: 1124-1128

27 Wilkins B J, Molkentin J D. Calcium-calcineurin signaling in the regulation of cardiac hypertrophy. Biochem Biophys Res Commun, 2004, 322: 1178-1191

28 Lin Z, Murtaza I, Wang K, et al. miR-23a functions downstream of NFATc3 to regulate cardiac hypertrophy. Proc Natl Acad Sci USA, 2009, 106: 12103-12108

29 Ikeda S, He A, Kong S W, et al. MicroRNA-1 negatively regulates expression of the hypertrophy-associated calmodulin and Mef2a genes. Mol Cell Biol, 2009, 29: 2193-2204

30 Da C M P, Salic K, Gladka M M, et al. MicroRNA-199b targets the nuclear kinase Dyrk1a in an auto-amplification loop promoting calcineurin/NFAT signalling. Nat Cell Biol, 2010, 12: 1220-1227

31 Care A, Catalucci D, Felicetti F, et al. MicroRNA-133 controls cardiac hypertrophy. Nat Med, 2007, 13: 613-618

32 van Rooij E, Sutherland L B, Qi X, et al. Control of stress-dependent cardiac growth and gene expression by a microRNA. Science, 2007, 316: 575-579

33 Callis T E, Pandya K, Seok H Y, et al. MicroRNA-208a is a regulator of cardiac hypertrophy and conduction in mice. J Clin Invest, 2009, 119: 2772-2786

34 Nishi H, Ono K, Horie T, et al. MicroRNA-27a regulates beta cardiac myosin heavy chain gene expression by targeting thyroid hormone receptor beta1 in neonatal rat ventricular myocytes. Mol Cell Biol, 2011, 31: 744-755

35 Ago T, Sadoshima J. Thioredoxin1 as a negative regulator of cardiac hypertrophy. Antioxid Redox Signal, 2007, 9: 679-687

36 Yang Y, Ago T, Zhai P, et al. Thioredoxin 1 negatively regulates angiotensin II-induced cardiac hypertrophy through upregulation of miR-98/let-7. Circ Res, 2011, 108: 305-313

37 Wu K H, Mo X M, Han Z C, et al. Stem cell engraftment and survival in the ischemic heart. Ann Thorac Surg, 2011, 92: 1917-1925

38 Mann D L, Bogaev R, Buckberg G D. Cardiac remodelling and myocardial recovery: lost in translation? Eur J Heart Fail, 2010, 12: 789-796

39 Ren X P, Wu J, Wang X, et al. MicroRNA-320 is involved in the regulation of cardiac ischemia/reperfusion injury by targeting heat-shock protein 20. Circulation, 2009, 119: 2357-2366

40 Zhang $\mathrm{X}$, Wang $\mathrm{X}$, Zhu $\mathrm{H}$, et al. Synergistic effects of the GATA-4-mediated miR-144/451 cluster in protection against simulated ischemia/reperfusion-induced cardiomyocyte death. J Mol Cell Cardiol, 2010, 49: 841-850

41 Dong S, Cheng Y, Yang J, et al. MicroRNA expression signature and the role of microRNA-21 in the early phase of acute myocardial infarction. J Biol Chem, 2009, 284: 29514-29525

$42 \mathrm{Xu} \mathrm{C}, \mathrm{Lu} \mathrm{Y,} \mathrm{Pan} \mathrm{Z,} \mathrm{et} \mathrm{al.} \mathrm{The} \mathrm{muscle-specific} \mathrm{microRNAs} \mathrm{miR-1} \mathrm{and}$ miR-133 produce opposing effects on apoptosis by targeting HSP60, HSP70 and caspase-9 in cardiomyocytes. J Cell Sci, 2007, 120: 3045-3052

43 Shan Z X, Lin Q X, Fu Y H, et al. Upregulated expression of miR-1/miR-206 in a rat model of myocardial infarction. Biochem Biophys Res Commun, 2009, 381: 597-601

44 Rowland R T, Meng X, Cleveland J C, et al. Cardioadaptation induced by cyclic ischemic preconditioning is mediated by translational regulation of de novo protein synthesis. J Surg Res, 1997, 71: $155-160$

45 Murry C E, Jennings R B, Reimer K A. Preconditioning with ischemia: a delay of lethal cell injury in ischemic myocardium. Circulation, 1986, 74: 1124-1136

46 Rane S, He M, Sayed D, et al. Downregulation of miR-199a derepresses hypoxia-inducible factor-1alpha and Sirtuin 1 and recapitulates hypoxia preconditioning in cardiac myocytes. Circ Res, 2009, 104: 879-886

47 Lim J H, Lee Y M, Chun Y S, et al. Sirtuin 1 modulates cellular responses to hypoxia by deacetylating hypoxia-inducible factor 1alpha. Mol Cell, 2010, 38: 864-878 
48 Qian L, Van Laake L W, Huang Y, et al. miR-24 inhibits apoptosis and represses Bim in mouse cardiomyocytes. J Exp Med, 2011, 208: 549-560

49 Wang J X, Jiao J Q, Li Q, et al. miR-499 regulates mitochondrial dynamics by targeting calcineurin and dynamin-related protein-1. Nat Med, 2011, 17: 71-78

50 Di Diego J M, Antzelevitch C. Ischemic ventricular arrhythmias: Experimental models and their clinical relevance. Heart Rhythm, 2011, 8: 1963-1968

51 Mill J G, Stefanon I, Dos S L, et al. Remodeling in the ischemic heart: the stepwise progression for heart. Braz J Med Biol Res, 2011, 44: 890-898

52 Yang B, Lin H, Xiao J, et al. The muscle-specific microRNA miR-1 regulates cardiac arrhythmogenic potential by targeting GJA1 and KCNJ2. Nat Med, 2007, 13: 486-491

53 Lerner D L, Yamada K A, Schuessler R B, et al. Accelerated onset and increased incidence of ventricular arrhythmias induced by ischemia in Cx43-deficient mice. Circulation, 2000, 101: 547-552

54 Plaster N M, Tawil R, Tristani-Firouzi M, et al. Mutations in Kir2.1 cause the developmental and episodic electrical phenotypes of Andersen's syndrome. Cell, 2001, 105: 511-519

55 Luo X, Lin H, Pan Z, et al. Down-regulation of miR-1/miR-133 contributes to re-expression of pacemaker channel genes $\mathrm{HCN} 2$ and HCN4 in hypertrophic heart. J Biol Chem, 2008, 283: 20045-20052

56 Michels G, Er F, Khan I, et al. Single-channel properties support a potential contribution of hyperpolarization-activated cyclic nucleotide-gated channels and If to cardiac arrhythmias. Circulation, 2005, 111: 399-404

57 Matkovich S J, Wang W, Tu Y, et al. MicroRNA-133a protects against myocardial fibrosis and modulates electrical repolarization without affecting hypertrophy in pressure-overloaded adult hearts. Circ Res, 2010, 106: 166-175

58 Lu Y, Zhang Y, Wang N, et al. MicroRNA-328 contributes to adverse electrical remodeling in atrial fibrillation. Circulation, 2010, 122: 2378-2387

59 Valadi H, Ekstrom K, Bossios A, et al. Exosome-mediated transfer of mRNAs and microRNAs is a novel mechanism of genetic exchange between cells. Nat Cell Biol, 2007, 9: 654-659

60 Hunter M P, Ismail N, Zhang X, et al. Detection of microRNA expression in human peripheral blood microvesicles. PLoS ONE, 2008, 3: e3694
61 Rabinowits G, Gercel-Taylor C, Day J M, et al. Exosomal microRNA: a diagnostic marker for lung cancer. Clin Lung Cancer, 2009, 10: 42-46

62 Camussi G, Deregibus M C, Bruno S, et al. Exosomes/microvesicles as a mechanism of cell-to-cell communication. Kidney Int, 2010, 78: 838-848

63 Kogure $\mathrm{T}$, Lin $\mathrm{W} \mathrm{L}$, Yan I K, et al. Intercellular nanovesicle-mediated microRNA transfer: A mechanism of environmental modulation of hepatocellular cancer cell growth. Hepatology, 2011, 54: $1237-1248$

64 Turchinovich A, Weiz L, Langheinz A, et al. Characterization of extracellular circulating microRNA. Nucleic Acids Res, 2011, 39: 7223-7233

65 Ji X, Takahashi R, Hiura Y, et al. Plasma miR-208 as a biomarker of myocardial injury. Clin Chem, 2009, 55: 1944-1949

66 Wang G K, Zhu J Q, Zhang J T, et al. Circulating microRNA: a novel potential biomarker for early diagnosis of acute myocardial infarction in humans. Eur Heart J, 2010, 31: 659-666

67 D'Alessandra Y, Devanna P, Limana F, et al. Circulating microRNAs are new and sensitive biomarkers of myocardial infarction. Eur Heart J, 2010, 31: 2765-2773

68 Ai J, Zhang R, Li Y, et al. Circulating microRNA-1 as a potential novel biomarker for acute myocardial infarction. Biochem Biophys Res Commun, 2010, 391: 73-77

69 Cheng Y, Tan N, Yang J, et al. A translational study of circulating cell-free microRNA-1 in acute myocardial infarction. Clin Sci (Lond), 2010, 119: 87-95

70 Li S, Zhu J, Zhang W, et al. Signature microRNA expression profile of essential hypertension and its novel link to human cytomegalovirus infection. Circulation, 2011, 124: 175-184

71 Corsten M F, Dennert R, Jochems S, et al. Circulating microRNA-208b and microRNA-499 reflect myocardial damage in cardiovascular disease. Circ Cardiovasc Genet, 2010, 3: 499-506

72 Cheng Y, Tan N, Yang J, et al. A translational study of circulating cell-free microRNA-1 in acute myocardial infarction. Clin Sci (Lond), 2010, 119: 87-95

73 Kuwabara Y, Ono K, Horie T, et al. Increased microRNA-1 and microRNA-133a levels in serum of patients with cardiovascular disease indicate myocardial damage. Circ Cardiovasc Genet, 2011, 4: $446-454$

Open Access This article is distributed under the terms of the Creative Commons Attribution License which permits any use, distribution, and reproduction in any medium, provided the original author(s) and source are credited. 